\title{
Challenges for Electron Tomography of Nanoscale Electrical Devices: Geometry and Beam Damage
}

\author{
Matthew Mecklenburg, ${ }^{* * *}$ Brian Shevitski, ${ }^{* * *}$ S. B. Singer,*** and B. C. Regan, ${ }^{* * *}$ \\ * Department of Physics and Astronomy, University of California, Los Angeles, California 90095 \\ ** California NanoSystems Institute, University of California, Los Angeles, California 90095
}

With the explosion of work on carbon nanostructures in the last 25 years, characterization techniques based on transmission electron microscopy (TEM) have become increasingly important. Accurate and effective characterization of such materials as carbon nanotubes and graphene requires a working knowledge of how the imaging technique interacts with, and may in fact modify, the sample. Recent improvements in electron optics, microscope stability and automation have made beam sensitivity the limiting factor in the imaging of these materials. We present here results from our study on the tomographic imaging and reconstruction of an electrical device utilizing a single multiwalled carbon nanotube as its active element.

Sample geometry is a problem unique to imaging nanoscale electrical devices. If multiwalled carbon nanotubes are the topic of interest, it would have been most straightforward to mount them on a standard TEM sample grid that can easily be tilted to large angles. In our case we sought to determine the three-dimensional structure of our nanoscopic lamp in its operational configuration and the range of accessible tilt angles was limited by the device geometry as defined by the silicon chip and its silicon nitride membrane window.

As a result of the many images that must be acquired, data acquisition for tomographic reconstruction exposes the sample to large and potentially damaging electron beam doses as compared to conventional TEM imaging, see FIG. 1. Accelerating voltages of $300 \mathrm{kV}$ and $200 \mathrm{kV}$ will degrade a nanotube sample on timescales on the order of seconds or minutes. This damage is ascribed to the knock-on or sputtering effect, where an electron colliding with a carbon atom ejects it from its position in the crystal lattice. Smith and Luzzi [1] found no evidence of damage to singlewalled carbon nanotubes after more than two hours of irradiation with an $80 \mathrm{keV}$ beam. Because carbon nanomaterials are relatively beam sensitive, little work has been done on, for instance, the tomographic reconstruction of individual carbon nanotubes. Tomography has been applied to study the interaction between small metal particles and carbon nanotubes with both few [2] and many [3] walls. More recent developments include three-dimensional reconstructions of $\mathrm{TiO}_{2}$ nanotubes grown on carbon nanotube templates [4], and ultrafast, time-resolved tomograms showing the vibrational modes of multiwalled carbon nanotubes [5]. To our knowledge tomography of an electronic device based on a carbon nanotube has not previously been demonstrated, our results are can be see in FIG. 2 .

References

[1] B. W. Smith, D. E. Luzzi, Electron irradiation effects in single wall carbon nanotubes, Journal of Applied Physics 90 (7) (2001) 3509-3515. 
[2] J. J. Cha, M. Weyland, J. F. Briere, I. P. Daykov, T. A. Arias, D. A. Muller, Threedimensional imaging of carbon nanotubes deformed by metal islands, Nano Letters 7 (12) (2007) 3770-3773.

[3] O. Ersen, J. Werckmann, M. Houlle, M. J. Ledoux, C. Pham-Huu, 3d electron microscopy study of metal particles inside multiwalled carbon nanotubes, Nano Letters 7 (7) (2007) 1898-1907.

[4] A. B. Hungria, D. Eder, A. H. Windle, P. A. Midgley, Visualization of the three-dimensional microstructure of tio2 nanotubes

by electron tomography, Catalysis Today 143 (3-4) (2009) 225-229.

[5] O. H. Kwon, A. H. Zewail, 4d electron tomography, Science 328 (5986) (2010) 1668-1673.

[6] P. Gilbert, Iterative methods for 3-dimensional reconstruction of an object from projections, Journal of Theoretical Biology 36 (1) (1972) 105-117.

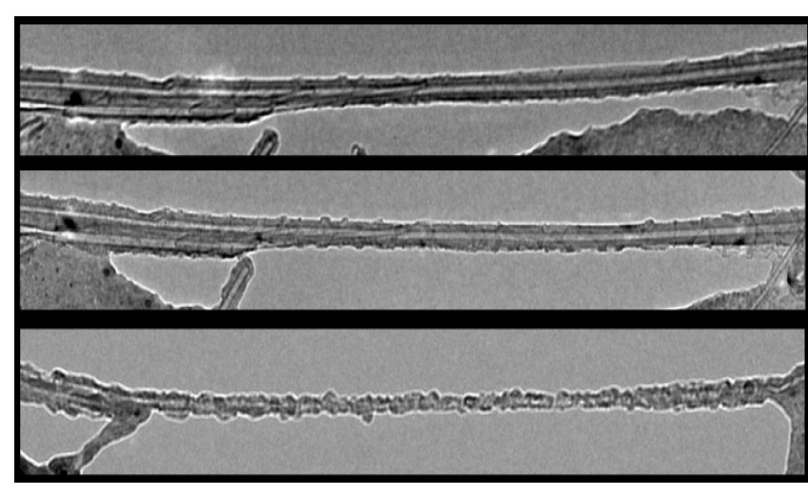

FIG. 1.

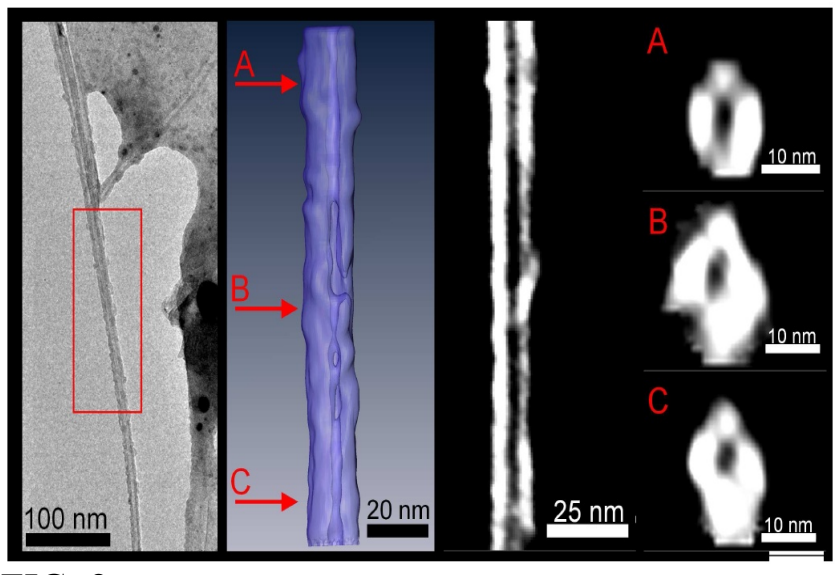

FIG. 2 .

FIG. 1. Low-magnification time series showing degradation of a $\sim 600 \mathrm{~nm}$ long nanotube macrostructure with increasing beam exposure. From top to bottom these images show the same nanotube section after receiving integrated electron doses of $10^{20}, 10^{21}$, and $10^{22}$ electrons $/ \mathrm{cm}^{2}$. Tomographic images were acquired at magnifications in the range from $6.3 \mathrm{kX}$ and $56 \mathrm{kX}$, with corresponding dose rates ranging from approximately 10 to 100 electrons $/ \AA^{2} / \mathrm{s}$. Even at an accelerating voltage of $80 \mathrm{kV}$ it is clear that a dose of $10^{22}$ electrons $/ \mathrm{cm}^{2}$, or roughly 11 hours of continuous imaging at $21 \mathrm{kX}$, is sufficient to remove roughly half of the material from a suspended multiwalled carbon nanotube.

FIG.2. Reconstruction of a multiwalled carbon nanotube. To generate the reconstruction, sequential images were aligned using band-pass filters and cross-correlation in FEI's Inspect3D. The tilt axis was then manually adjusted to be along the long axis of the nanotube segment to be reconstructed. Reconstructions based on weighted back projection were used to evaluate the quality of the alignment. This process was iterated until there was no noticeable change in image alignment or reconstruction quality. Inspect3D's implementation of SIRT (Simultaneous Iterative Reconstruction Technique [6]) was used to reconstruct the final volume. Finally, the Amira software package was used for data segmentation, surface rendering, and graphics generation. 